\title{
An Approach to Automatic Target Recognition in Radar Images Using SVM
}

\author{
Noslen Hernández ${ }^{1}$, José Luis Gil Rodríguez ${ }^{1}$, Jorge A. Martin², \\ Francisco Silva Mata ${ }^{1}$, Ricardo González ${ }^{1}$, and Raúl Álvarez ${ }^{2}$ \\ ${ }^{1}$ Advanced Technologies Application Center, MIMBAS \\ 7a \#21812 e/ 218 y 222, Rpto. Siboney, Playa. C.P. 12200 \\ Ciudad de la Habana, Cuba \\ Office Phone number: (+) 537.271.4787; Fax number: (+) 537.273.0045 \\ \{nhernandez, jlgil, fjsilva, rgazapo\}@cenatav.co.cu \\ http: / / www. cenatav.co.cu \\ 2 “Granma” Navy Academy, Micro X, Alamar, Habana del Este, \\ Ciudad de La Habana \\ Fax: 939676
}

\begin{abstract}
This paper introduces an Automatic Target Recognition (ATR) method based on X Band Radar image processing. A software which implements this method was developed following four principal stages: digital image formation, image preprocessing, feature selection through a combination of C4.5 Decision Tree and PCA and classification using SVM. The automatic process was validated using two images sets, one of them containing real images with natural noise levels and the other with different degrees of impulsive noise contamination. The method achieves a very nice computation behavior and effectiveness, high accuracy and robustness in noise environments with a low storage memory and high decision speed.
\end{abstract}

Keywords: target recognition, X Band radar, SVM, image processing, feature selection.

\section{Introduction}

$\mathrm{X}$-band radar systems have been of great interest in the last few decades. The relative short wavelength at $\mathrm{X}$-band frequencies (8-12 GHz) makes possible the obtaining of high-resolution radars (HRR) images for target identification and discrimination [1]. Some civil, military and government institutions often use X-band radar systems for: maritime and air traffic control, defense tracking, weather monitoring, and others applications. Target recognition is a challenging problem intensified on the extraction of target features from the available raw data as the recognition performance is strongly determined by the informative power of the features [2]. Furthermore, the objects feature extraction is an especially difficult problem highly dependent on three aspects: Radar Cross Section $\left(\mathrm{RCS}^{1}\right)$ of targets; image formation process and particularly on the image preprocessing sequence applied. Target detection on the sea, under some abnormal conditions, is possible with an image of the sea surface. This

\footnotetext{
${ }^{1} \mathrm{RCS}$ is "a measure of the reflective strength of a radar target" [3].
} 
image is created with information given by the standard marine $\mathrm{X}$-band radar. In these images we can visualize the changes in the wave behavior produced by the presence of objects as sea clutter, clouds or ships in sailing. The automatic recognition of ships navigating is the main purpose of this work.

This paper introduces an automatic target recognition (ATR) method based on X Band Radar image processing and it is organized as follows: digital image formation, image preprocessing, feature extraction and feature selection through a combination of C4.5 Decision Tree and PCA and classification using SVM. The automatic process was validated using two images sets, one of them containing real images with natural noise levels and the other with different levels of impulsive noise contamination. The method achieves a high accuracy in the classification process, permitting a good identification of the target of concern.

\section{Radar Image Formation and Preprocessing}

The formation of a radar image takes place starting from the digitization of the captured echoes when the electromagnetic signal emitted by the radar is reflected by the objects. The reception of the echoes depends on the maritime object characteristics, in particular on its RCS. The reception of the echoes also depends on the radar technical characteristics as: the power of transmitted signal, impulse time duration, width of the signal band and the antenna gain.

Received echoes digitization is obtained through a specialized acquisition hardware. It has an analog/digital converter used as the communication interface between radar and computer. The echoes are one-dimensional electromagnetic signals synchronized in time and also in exploration angles. At each angle, a one-dimensional signal is captured by the sensor during its rotation. Its digitized values are converted into a two-dimensional representation [4] from where an image is formed and whose intensity levels are normalized in the interval $[0 . .255]$.

The RCS of maritime targets is expressed in square meters $\left(\mathrm{m}^{2}\right)$. Generalized values of RCS for a variety of ships are given in [5]. Taking averages values of RCS a division in small targets $\left(<500 \mathrm{~m}^{2}\right)$, medium targets $\left(500\right.$ to $\left.2000 \mathrm{~m}^{2}\right)$ and big targets $\left(>2000 \mathrm{~m}^{2}\right)$ was made. Present research attention focus was put on big targets in 15 miles scale.

The main objective of image preprocessing stage is to prepare the image for the automatic analysis, which includes the reduction of the impulsive noise, and some objects that are not of interest (sea clutter) applying a $3 \times 3$ pixels median filter and a $3 \times 3$ pixels high pass filter [6]. The next step is the image binarization in order to achieve the objects (target/no target) segmentation applying an automatic threshold algorithm following the iterative procedure proposed by González and Woods in [7].

\section{Feature Extraction and Selection}

The next step is to represent and describe these objects in a form suitable for further computer processing. A representation with 11 boundary and region descriptors was 


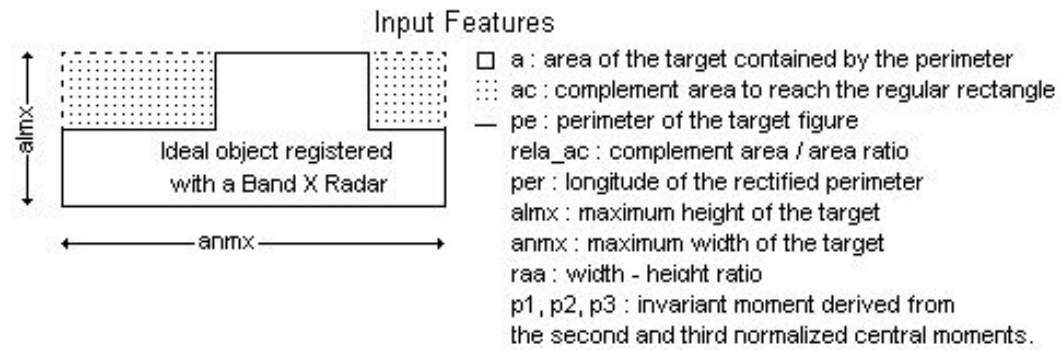

Fig. 1. Graphical representation of the 11 features in an ideal object present in a preprocessed $X$ Band Radar image

chosen using a Geometric and Topologic Model [8] [9]. Fig. 1 shows the full feature set designed for the textural characterization of objects.

To known the most significant features to describe the Targets (object of interest), C4.5 Decision Tree and Principal Component Analysis (PCA) were used. Both techniques were applied separately on a set of samples, described by original features, composed by objects from different images in order to guarantee a high representativeness. We will refer to this set as "S", which contains 470 objects, 178 in the Target class and 292 in the No Target class.

\subsection{C4.5 Decision Tree Used for Feature Selection}

In general, decision trees represent a disjunction of conjunctions of constraints on the attribute-values of examples. The selection is based on a statistical property called information gain that measures how well a given attribute separates the training examples according to their classification. In this sense the tree' nodes will contain the more relevant features, being the more important feature located at the high levels of tree [10].

An instance is classified by starting at the root node of the decision tree, testing the attribute specified by this node and moving down by the tree branch corresponding to the value of the attribute. This process is then repeated at the node on this branch and so on, until a leaf node is reached which provides the classification of the instance.

Three different experiments were designed and applied to the set "S" (Fig. 2). In the Experiment 1, "S" was randomly divided in four disjoint training sets which have the same number of samples and also a good balance among target and no target ones. Four decision trees were built; each of them validated using 10 folds Cross Validation. In the Experiment 2, four different sets were prepared using resampling on "S"; each of them was divided in $75 \%$ of the samples for training and the other $25 \%$ for testing. This means that each classifier was built with training sets that could be contain common samples. In the Experiment 3, only one decision tree was built using the set "S" as training set and it was validated with 10 folds Cross Validation. 
Results show that some of the initial features do not appear in any of the trees. This mean that they are not relevant for targets characterization and recognizing and it is possible to eliminate them. Finally were selected $\mathbf{a}, \mathbf{a c}, \mathbf{p e}, \mathbf{p 1}, \mathbf{p 2}$, per as a result of the union of the partial results of each decision tree.

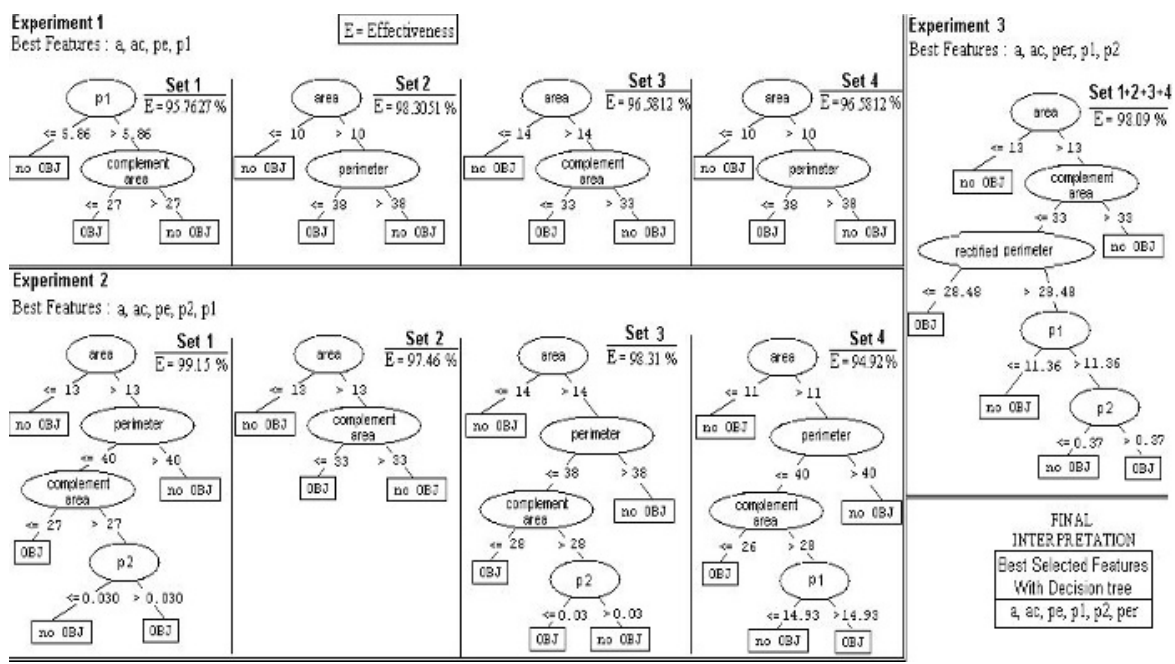

Fig. 2. Features selected with C4.5 Decision Tree

\subsection{PCA Used for Feature Selection}

PCA is a powerful tool used in exploratory data analysis. It provides a way to reduce the dimensionality of the data finding linear combinations of the original features which account for maximal amounts of variation, known as principal components or eigenvectors. PCA eigenvectors have several desirable properties. First, they are mutually orthogonal, which is the same as saying they are uncorrelated. Second, eigenvectors can be computed in order of decreasing variance. Thus, the first eigenvector accounts for the maximum amount of variance and each successive eigenvector accounts for less of the remaining variance in the data [11].

PCA is based on the idea of expressing a matrix $\mathbf{X}$ as the product of two other matrices, the scores matrix $\mathbf{T}$ and the transpose of the loadings matrix $\mathbf{L}: X=T L^{T}$. If only the first $\mathrm{k}$ columns of the scores and loadings matrices are retained, then $X \approx \hat{X}=T_{k} L_{k}^{T}$. The concentration of variance in the first several PCs permits the omission of later factors without significant loss of information, so the transformed and preprocessed data matrix is imperfectly reconstructed from the trimmed scores and loadings, that is, within some residual error $E_{k}=X-T_{k} L_{k}^{T}$.

PCA has been done in a set of samples "S". With the aim to give the same importance to all features we scale our data before doing PCA to zero mean and unit variance. From the analysis of the correlation matrix obtained with PCA and some plots of features against features we conclude that the features pe and per are highly correlated. 
To reduce the dimensionality of the data implies that it contain irrelevant or random variation, some of which can be removed by retaining only the principal components that capture relevant information. Only the first three principal components which explain $94.14 \%$ of world variance were retained, as we can see in Fig. 3.

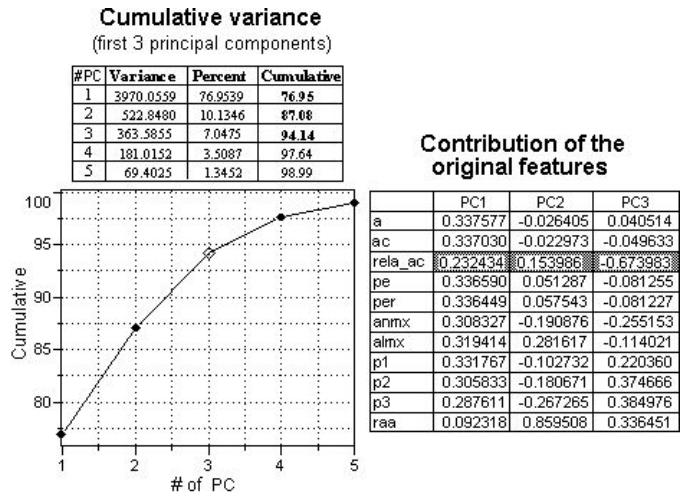

Fig. 3. Cumulative variance versus principal components and contribution of the original features to the first three components

The contribution of the original features to the first three principal components is shown in the same figure. All the original variables contribute in a significant manner to any one of the $\mathrm{PC}_{\mathrm{s}}$. For this reason none of them can be removed in this step. In order to look for the relevant features is necessary to obtain the modeling power of them.

\section{Modeling Power}

Modeling Power varies with the number $\mathrm{k}$ of principal component selected $(\mathrm{k}=3$ in our case) but is variable-oriented. Typically, it is not helpful in determining the optimal number of factors to retain, but does point out important variables.

Modeling Power of a variable is defined as:

$$
M P_{j}=1-\frac{\hat{S}_{j}}{S_{0 j}}
$$

where $\hat{S}_{j}$ is the root square of variable residual variance $\hat{S}_{j}^{2}$, which is calculated using the $\mathrm{j}^{\text {th }}$ column of the residual matrix $E_{k}$ defined above:

$$
S_{j}^{2}=\frac{e_{j} e_{j}^{T}}{n-k-1}
$$

and $S_{0 j}$ is the root square of the total variance of that variable calculated as: 


$$
\hat{S}_{0 j}^{2}=\frac{1}{n-1} \sum_{i}^{n}\left(x_{i j}-\bar{x}_{j}\right)^{2}
$$

When the power of a variable to model information in the data increases, MP approaches 1; contrary when it decreases, MP approaches 0 . Even with random data, some features will exhibit high modeling power, so an absolute threshold cannot be specified. Instead, the different variables should be compared based on their relative modeling power.

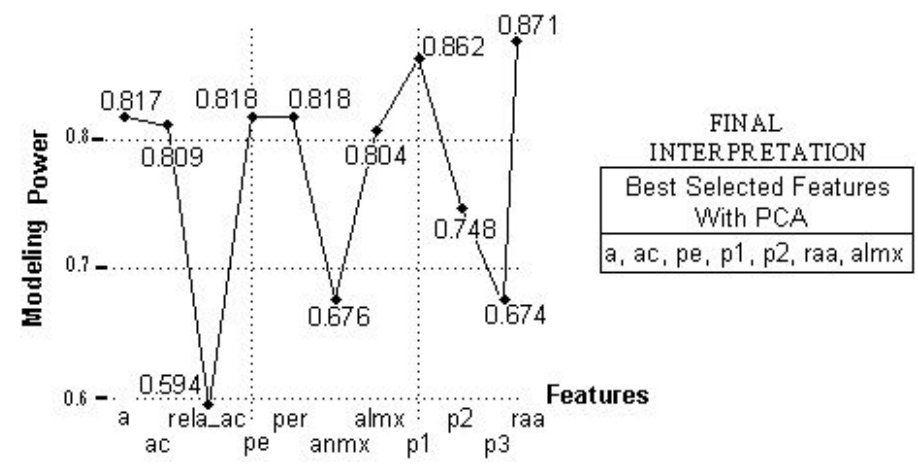

Fig. 4. Modeling power versus features

Comparing the 11 original features by their modeling power value (Fig. 4) is possible to conclude that features a, ac, pe, per, p1, p2, raa, almx have the highest values of discriminator power.

As we can notice there are interceptions between features selected as relevant by both techniques. For final feature selection, a combined interpretation of the results was made and the union of the features selected as relevant by both methods was used as final criterion. Taking into account the high correlation that exist between pe and per it is necessary to reject one of them. The selection of pe instead of per was based in the fact that pe appears more times in the experiments realized with Decision Trees.

The features selected (a, ac, pe, p1, p2, raa, almx) were used to build an automatic classifier.

\section{Classification}

In our case a binary classification problem (Target and No Target objects) is necessary to solve. One of the relatively new and promising methods for learning separating functions in pattern recognition (classification) tasks are the Support Vector Machines (SVM) developed by Vapnik and Chervonenkis [12] [13] [14] [15].

In a SVM learning for two linearly separable classes, one aims at finding a separating "maximal margin" hyperplane which gives the smallest generalization error among the infinite number of possible hyperplanes [12]. The data on margin 
and/or the closest ones are called support vectors. They are found by solving a quadratic programming (QP) problem. Very often the separation function between the classes is nonlinear. In this case, the data will be mapped from an input space into a high dimensional feature space by a nonlinear transformation $\phi(x)$. Because the $\mathrm{QP}$ problem in a feature space depends only on a dot product $\phi\left(x_{i}\right)^{T} \phi\left(x_{j}\right)$ the every learning can be performed by using Mercer theorem [13] for positive definite functions that allows replacement of $\phi\left(x_{i}\right)^{T} \phi\left(x_{j}\right)$ by a positive definite symmetric kernel function $K\left(x_{i}, x_{j}\right)=\phi\left(x_{i}\right)^{T} \phi\left(x_{j}\right)$. In our case a non-linear SVM with a Gaussian radial basis function (RBF) kernel [13] was used.

The QP optimization problem also have the convex properties and hence not local minima, given us a unique and optimal solution.

There are two basic design parameters that determine the goodness of an SVM Classifier. Here, they are, $\mathrm{C}$ which determines a tradeoff between maximizing the margin and minimizing the numbers of errors and $\gamma$ that define the width of 7 dimensional Gaussian functions contained on the diagonal of the covariance matrix. Both parameters can be selected performing a "grid-search" using $\mathrm{k}$ folds crossvalidation. Basically, pairs of $(\mathrm{C}, \gamma)$ values are tested and the one with the best crossvalidation accuracy is picked.

The used of grid search is recommended when we may not feel safe to use methods which avoid doing an exhaustive parameter search by approximations or heuristics.

\section{Results and Discussion}

SVM were training with data given as vectors $(x, y)$, where $x$ is a sevendimensional input vector of values of selected features, i.e., vector $x \in \mathfrak{R}^{7}$, the desired value $y$ is a binary value variable, i.e., $y \in\{+1,-1\}$ for Target and No Target objects respectively. The set " $\mathrm{S}$ " defined in section 3 was used to train.

In order to obtain the best parameters value to train the SVM a grid search using 10 folds Cross Validation was done. The ranges assigned to each hyperparameter were $\log _{2} \mathrm{C}=(-5,-4, \ldots, 14,15)$ and $\log _{2} \gamma=(-15,-14, \ldots, 4)$. Then, with the values obtained by

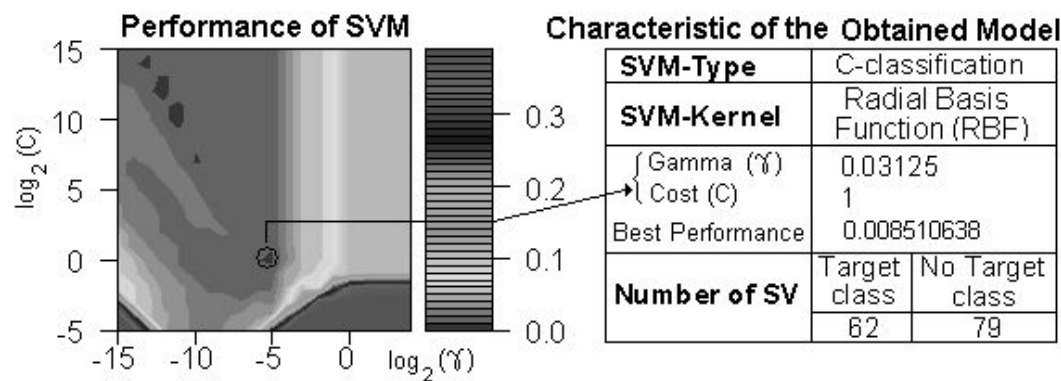

Fig. 5. Left: The best pair of parameter $(C, \gamma)$ values was extracted from the area within the circle marked. Right: The general characteristic of the obtained model are presented. 
grid search, the SVM Classifier was trained again and the model was validated using 10 folds Cross Validation, giving us an accuracy of $99.15 \%$.

The accuracy of SVM trained using different training parameters combination and the characteristic of the obtained model are shown in Fig. 5.

In order to SVM training and classification process was used the LIBSVM library [16].

In processed $\mathrm{X}$ Band radar images there are much more No Target objects than Target objects. In learning extremely imbalanced data, the overall classification accuracy is often not an appropriate measure of performance. A trivial classifier that predicts every case as the majority class can still achieve very high accuracy. We use metrics such as true negative rate, true positive rate, G-mean and Precision [17] to evaluate the performance of our learning algorithms on imbalanced data. These metrics are functions of the confusion matrix [17]. Different test sets were prepared in order to obtain statistics about the performance of the automatic method proposed. The process was applied repeatedly on each image belonging to these sets.

\subsection{Performance with Real Images}

In this experiment, a set of $27 \mathrm{X}$ Band radar real images never employees during the training process were used as validation set. These images were collected during six months. They were processed using our method and the confusion matrix and metrics are shown in Fig. 6.

\begin{tabular}{|c|c|c|c|c|}
\hline & & \multicolumn{2}{|c|}{ Predicted Class } & \\
\hline & & Targets & No Targets & True Positive Rate $\left(\mathrm{Acc}^{+}\right)=\frac{\mathrm{TP}}{\mathrm{TP}+\mathrm{FN}}=94.87 \%$ \\
\hline$\frac{\mathscr{Q}}{0}$ & Targets & $\begin{array}{c}\text { TP }=74 \\
\text { (True Positives) }\end{array}$ & $\begin{array}{l}\qquad \mathrm{FN}=4 \\
\text { (False Negatives) }\end{array}$ & 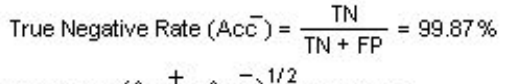 \\
\hline 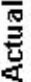 & No Targets & $\begin{array}{c}\text { FP }=12 \\
\text { (False Positives) }\end{array}$ & $\begin{array}{c}\mathrm{TN}=9228 \\
\text { (True Negatives) }\end{array}$ & Precision $=\frac{T P}{T P+F P}=86.05 \%$ \\
\hline
\end{tabular}

Fig. 6. Confusion Matrix and metrics calculated

The good results obtained in the classification task demonstrated the advantages attributed in the literature to the SVM as a two class classifier. The complete automatic process of an image was very fast, $3 \mathrm{sec}$ by image approximately, doing this method very good and robust for a real time detection of maritime targets.

\subsection{Performance with Artificial Noisy Images}

In this experiment, the principal objective was investigated the performance of our method at different levels of signal-to-ratio (SNR) noise. An arbitrary X Band Radar image containing all kind of targets and not targets was chosen. A set of noisy images were synthesized adding Salt and Pepper noise with levels of 0.01, 0.02, 0.03, 0.04 and 0.05 noise density $(\mathrm{dB})$. Table 1 presents the accuracy of the automatic method in noisy test conditions. 
Table 1. Results of our method in a set of noisy image at different levels of noise

\begin{tabular}{|c|c|c|c|c|c|c|}
\hline & \multirow{2}{*}{$\begin{array}{c}\text { Original } \\
\text { Image }\end{array}$} & \multicolumn{5}{|c|}{ Original Image + Salt \& Pepper Noise Density } \\
\cline { 3 - 7 } & & $\mathbf{0 . 0 1}$ & $\mathbf{0 . 0 2}$ & $\mathbf{0 . 0 3}$ & $\mathbf{0 . 0 4}$ & $\mathbf{0 . 0 5}$ \\
\hline True positive (\%) & 100 & 99.08 & 96.33 & 93.58 & 84.40 & 85.32 \\
\hline True negative (\%) & 98.55 & 98.27 & 98.19 & 98.11 & 98.01 & 97.93 \\
\hline $\begin{array}{c}\text { G-mean } \\
\text { Noise Level } \\
\text { Tolerance }\end{array}$ & 99.27 & 98.67 & 97.25 & 95.81 & 90.95 & 91.40 \\
\hline & OK & OK & OK & No OK & No OK \\
\hline
\end{tabular}

We can notice that our method has robustness in noisy images, staying above $90 \%$ for all tested levels of noise. However, only up to 0.03 noisy density levels were accepted as a consequence of the risk that implies a failure in the detection of Target objects. It is important to point out that these levels of not accepted noise are hardly found in real images after preprocessing stage.

Fig.7 shows a set of images representatives of all the process. After the final results are obtained, the user has the possibility of change any wrong response given by the automatic process.
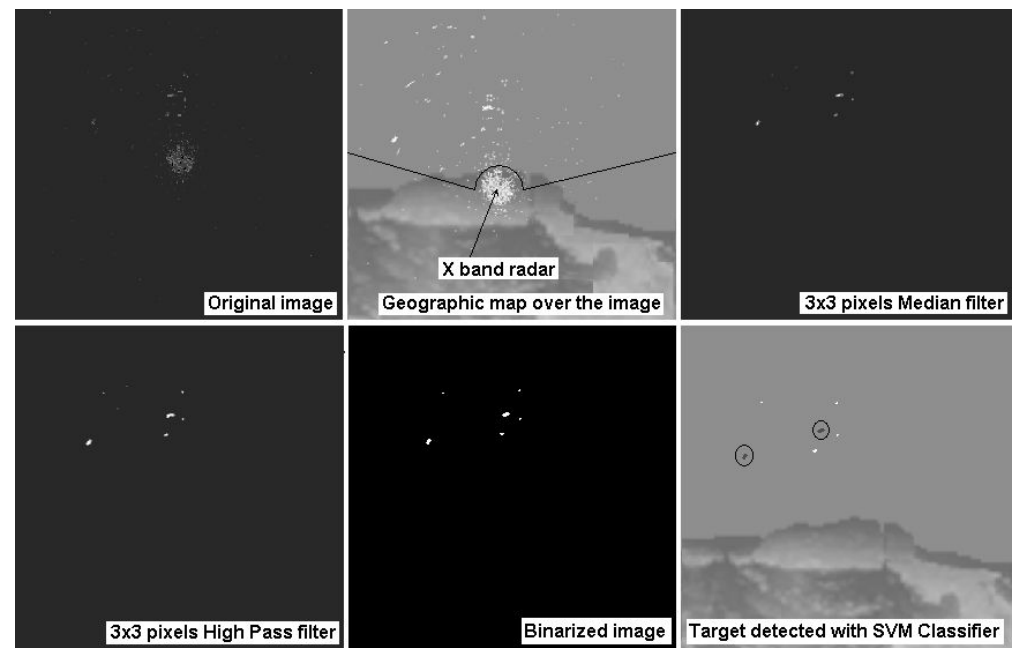

Fig. 7. Process for marine targets automatic recognition using $\mathrm{X}$ band radar sensor

\section{Conclusions and Future Work}

In this paper, the main concern was to obtain a novel and efficient approach for automatic target recognition in $\mathrm{X}$ band radar images. Its usefulness in two image sets, one of them with real image containing natural level of noise, and the other with noisy images at different levels of impulsive noise contamination was verified. We also 
applied successfully a combination of PCA and C4.5 Decision Tree to find out the relevant features among all features calculated by us.

The experimental results show that this method has a very good computational behavior and provide a very useful tool for real time ATR systems due to its simplicity and high decision speed.

We consider necessary to experiment with new king of features specially obtained from the frequency domain of the image.

\section{References}

1. Gallego, J., Pérez, F.: Detección de Blancos Extensos en Entornos Marinos y Terrestres Basada en Descriptores. Available in Internet at http://w3.iec.csic.es/URSI/ articulos_gandia_2005/articulos/RA/334.pdf. Date: May 14, 2006.

2. Turhan-Sayan, G.: Real Time Electromagnetic Target Classification Using a Novel Feature Extraction Technique with PCA-Based Fusion. IEEE Trans. on Antennas and Propagation, Vol. 53, No. 2, February 2005.

3. Bairachefski A. M., Sistemas de radiolocalización naval, Editora Transporte, Moscú, 1982.

4. Shinozawa, et al.: A weather radar image prediction method in local parallel computation. International Conference on Neural Networks, 1994.

5. Skolnik, M. I. (1980). Introduction to Radar Systems. McGraw-Hill Book Company, New York, 2. Ausgabe, 1563 pp.

6. Martin, J.A. y J.L. Gil: Cálculo de la navegación de altura empleando señales de posicionamiento global e imágenes meteorológicas. IPEN Journal, No. 29, Published by Pan American Institute of Naval Engineering, ISSN - 1011 - 5951. (2005).

7. Rafael C. González, and Richard E. Woods, "Digital Image Proccesing", 2nd ed. AddisonWesley Publishing Company, 2002.

8. Gil, J.L.: Estado actual de la representación y análisis de textura en imágenes (inédito). (2005).

9. Silva, F.J.: Estado actual de la representación y análisis de formas. (inédito). (2005).

10. Quinlan, R. J.: C4.5: Programs for Machine Learnig (Morgan Kaufmann Series in Machine Learning). Paperback- January 15. (1993).

11. Jackson, J.E.; A User's Guide to Principal Components, (John Wiley \& Sons: New York, 1991).

12. Vapnik, V.: The Nature of Statistical Learning Theory. New York: Springer Verlag. (1995).

13. Scholkopf, C., Burges, J., Smola, A.: Advances in Kernel methods: Support Vector Learning.MIT. Press. (1999).

14. Cristianini, Shawe-Taylor, J.: An introduction to Support Vector Machine. Cambridge University Press. (2000).

15. Burges, C. J. C.: A Tutorial on Support Vector Machines for Pattern Recognition. Data Mining and Knowledge Discovery 2(2): 121-167. (1999).

16. Chih-Chung Chang and Chih-Jen Lin: LIBSVM: A Library for Support Vector Machines, Dpt. Computer Science and Information Engineering, National Taiwan University. (2003). Available in Internet at http://www.csie.ntu.edu.tw/ cjlin/libsvm/. Date: May 14, 2006.

17. Computer Science 831: Knowledge Discovery in Databases. Dept. of Computer Science. University of Regina. Canada. Available in Internet at http://www2.cs.uregina.ca/ dbd/ cs831 /index.html. Date: May 15, 2006. 